\title{
State of Charge (SOC)-Based Active Power Sharing Method for Distributed Generations in an Islanded Microgrid
}

\author{
Yun-Su Kim and Seung-Il Moon \\ School of electrical and computer engineering \\ Seoul National University \\ Seoul, South Korea \\ yskim@powerlab.snu.ac.kr; moonsi@plaza.snu.ac.kr
}

\author{
Eung-Sang Kim \\ Smart Distribution Research Center \\ Korea Electrotechnology Research Institute \\ Changwon, South Korea \\ eskim@keri.re.kr
}

\begin{abstract}
Typically, a frequency droop control has been employed for active power sharing which inevitably causes the frequency deviation. In this paper, we propose an active power sharing method based on the state of charge (SOC) of battery energy storage system (BESS) instead of the frequency. In a similar concept of conventional primary and secondary frequency control, primary and secondary SOC control is developed for maintaining the frequency at the nominal value as well as the SOC at the desired level. The SOC information is assumed to be transmitted via communication infrastructure hence the communication time delay is considered. To enhance reliability, controllers of distributed generation (DG) units are designed to take account of the failure of communication system. The proposed method was modeled and simulated by MATLAB/SimPowerSystems to prove its effectiveness.
\end{abstract}

Keywords-active power sharing; battery energy storage system; distributed generation; islanded microgrid; state of charge.

\section{INTRODUCTION}

The electrical energy demand has been continuously grown recently [1] whereas the amount of supply has been gradually saturated to the limits on account of environmental, social, and geographical problems. On the other hand, fortunately, as distributed generations (DGs) have been increased [2], the microgrid was introduced as the solution for the power demand and supply balancing problem. The microgrid concept was first introduced in [3] and [4]. It is a low and medium voltage distribution network which is composed of DGs, energy storage devices, controllable loads, static switch, and etc. The microgrid has an ability to be connected to or isolated from the main utility by closing or opening the static switch at the point of common coupling (PCC). In grid-connected mode, the microgrid can be seen as a controllable load in the perspective of the main utility. In islanded mode, it can be continuously operated even in abnormal situations of the main utility such as faults or maintenance, thereby enhancing the system reliability. This characteristic gives a great advantage to an islanded microgrid. However, since the system inertia is low and the grid is weak, the frequency and voltage problems are bound to be occurred due to instantaneous load change or intermittent output active power of renewable energy systems such as wind and PV power. Among both frequency and voltage problems, we will focus on the frequency problem in this paper.

In conventional power systems, maintaining the system frequency at the nominal value has direct bearing on the power demand and supply balance. The active power sharing is facilitated based on the deviation of the system frequency. The power sharing methods in an islanded microgrid can be categorized based on whether or not they employ the droop control [5]. Most of them adopt the frequency droop control that is used in the conventional power system composed of large synchronous generators [6]-[9]. However, since an islanded microgrid has low system inertia, the frequency is likely to be largely deviated from its nominal value. As opposed to the conventional method, [10] and [11] proposed active and reactive power sharing based on voltage and frequency deviation, respectively. However, they are limited to be adopted to low voltage network only. Moreover, reactive power sharing is based on the frequency thereby the frequency deviation cannot be prevented. In [12], non-droopbased power sharing method using the communication infrastructure was proposed. However, the frequency deviation cannot be prevented if the load change occurs due to communication time delay. Reference [13] and [14] also proposed a centralized control method. However, they still employed the droop control thereby the frequency deviation is inevitable.

In this paper, we mainly focused on preventing the frequency deviation from its nominal value while the load change occurs in an islanded microgrid which has low system inertia. To this end, an inverter-interfaced battery energy storage system (BESS) is employed to constantly maintain the system frequency and voltage. The controllable distributed generation units are adjusted to maintain the state of charge (SOC) of the BESS at a certain level and to share active power based on the SOC deviation from pre-specified value. The active power sharing DG units are categorized as the primary and secondary SOC control units as similar as the conventional primary and secondary frequency control units. To enhance the system reliability, the frequency droop control is also added at the active power controller of DG units. The proposed method was modeled and simulated by MATLAB/SimPowerSystems.

The rest of this paper is organized as follows. Section II shows the system configuration and modeling. Section III illustrates the SOC-based active power sharing method. 


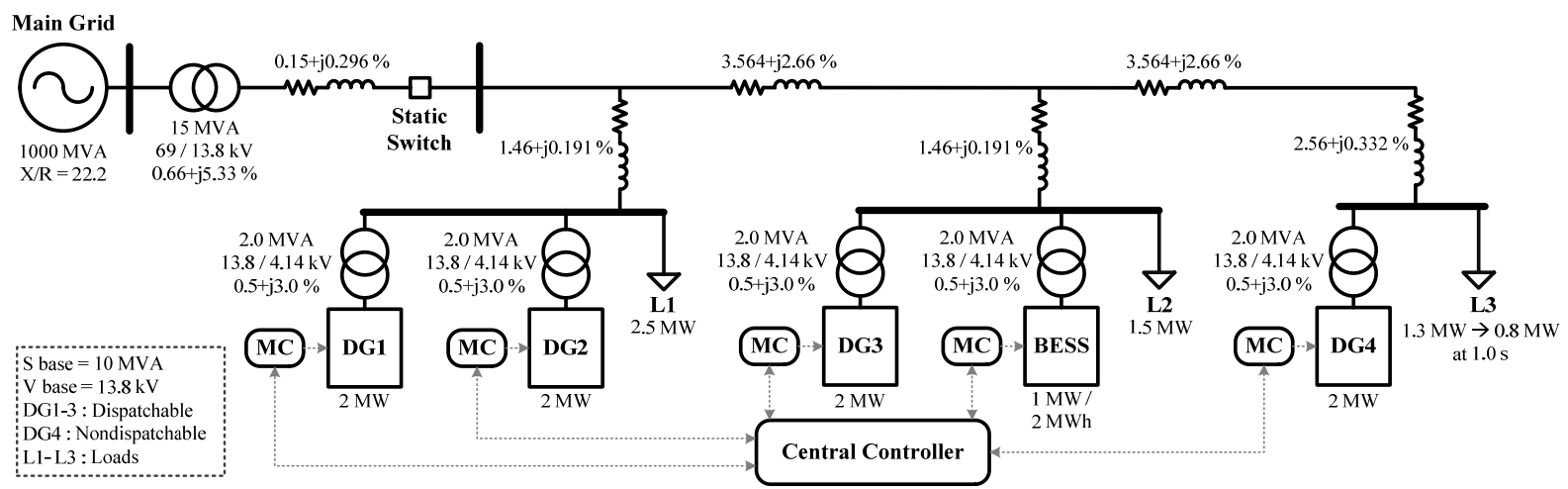

Figure 3. The microgrid configuration.

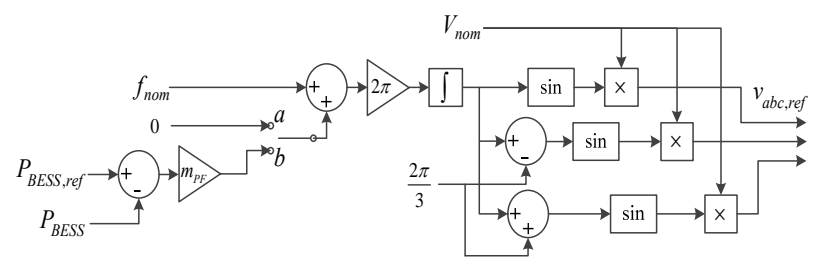

Figure 1. Control scheme of the BESS.

Section IV shows the simulation results and Section V outlines the conclusion.

\section{SyStem MODELING AND CONFIGURATION}

Fig. 1 shows the microgrid configuration which is referred from [15] and [16] with slight modification in the line connections and parameters. It is composed of the static switch, four 2-MW DG units, one 1-MW/2-MWh BESS, three loads and the microgrid central controller (MGCC) which exchanges the data including the SOC level with the microsource controller (MC).

Since we only consider islanded mode operation, the static switch is assumed to be opened. Three DG units (DG1-DG3) are controllable units and the other (DG4) is assumed as uncontrollable unit. Since we consider neither inverter switching dynamics nor harmonics, we assume inverters in the DG units and the BESS as ideal sources. Since the BESS is controlled as to maintain system frequency and voltage constantly, it is modeled with ideal voltage source. The other DG units are controlled as to adjust active and reactive power, so they are modeled with ideal current sources. The MGCC and MC are used to exchange the data, especially the SOC level of the BESS.

\section{SOC-BASED ACTIVE POWER SHARING METHOD}

\section{A. The BESS Control}

Fig. 2 shows the BESS controller where $f_{\text {nom }}$ is the nominal frequency, $V_{\text {nom }}$ is the nominal voltage magnitude, $P_{B E S S}$ and $P_{B E S S, r e f}$ are the active power output of the BESS and its reference value, respectively, $m_{P F}$ is the frequency droop coefficient, and $v_{a b c, \text { ref }}$ is the reference value of 3-phase voltage goes into the ideal voltage source. In normal

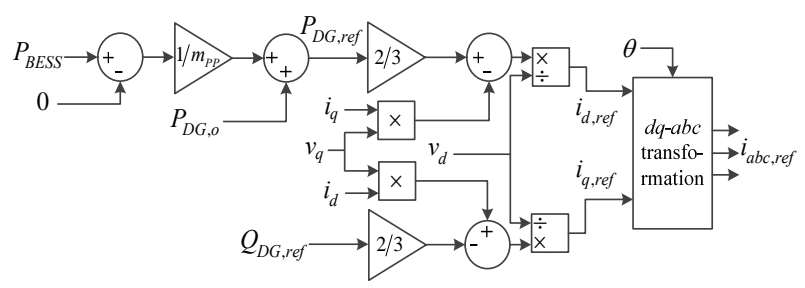

Figure 2. Control scheme of primary SOC control DG unit.

condition, switch is connected to node $a$, whereas in abnormal condition such as the communication system failure, it is connected to node $b$. In this way, the BESS is operated to form constant frequency in normal condition. If the communication system fails, the current SOC level of the BESS cannot be delivered to other DG units. To enhance the system reliability, in this case, the switch is connected to node $b$ thereby the BESS is operated as same as the conventional frequency droop control mode. However, the BESS has a limited capacity which leads the BESS unable to operate consistently while it becomes fully discharged. To overcome this problem, other controllable DG units are subjected to be controlled to maintain the SOC at the certain level and to share the active power of the system. These types of DG units are categorized as primary and secondary SOC control units as similar as the conventional primary and secondary frequency control units.

\section{B. Primary SOC Control}

The control scheme of the primary SOC control DG unit is shown in Fig. 3 where $m_{P P}$ is the droop coefficient, $P_{D G, o}$ is the dispatched active power reference, $P_{D G, r e f}$ and $Q_{D G, r e f}$ are the active and reactive power reference, respectively, $v_{d}$ and $v_{q}$ are $d$ - and $q$-component output voltage, respectively, $i_{d}$ and $i_{q}$ are $d$ - and $q$-component output current, respectively, $i_{d, \text { ref }}$ and $i_{q, \text { ref }}$ are their reference values, $\theta$ is the reference angle of $d$ - and $q$-axis, and $i_{a b c, \text { ref }}$ is the reference value of 3 phase current goes into the ideal current source. Since the active and reactive power can be expressed as

$$
\begin{aligned}
& P=3 / 2\left(v_{d} i_{d}+v_{q} i_{q}\right) \\
& Q=3 / 2\left(v_{q} i_{d}-v_{d} i_{q}\right),
\end{aligned}
$$




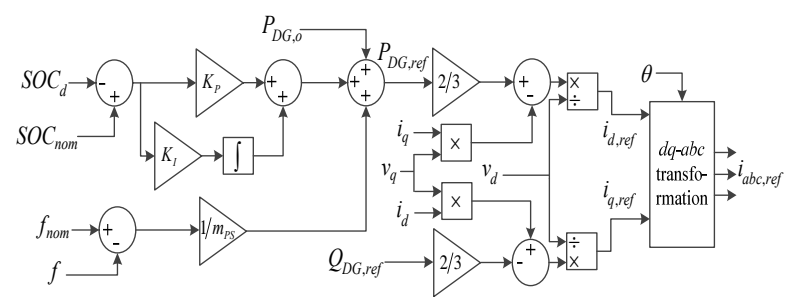

Figure 4. Control scheme of secondary SOC control DG unit.

$i_{d, r e f}$ and $i_{q, r e f}$ can be rearranged as

$$
\begin{aligned}
& i_{d, r e f}=\left(2 / 3 P_{D G, r e f}-v_{q} i_{q}\right) / v_{d} \\
& i_{q, r e f}=\left(v_{q} i_{d}-2 / 3 Q_{D G, r e f}\right) / v_{d} .
\end{aligned}
$$

Therefore, the reference $d$ - and $q$-component currents can be controlled as shown in Fig. 3 as well as Fig. 4.

To maintain the SOC at the certain level, it is important for the BESS to keep its output at 0. Hence, the main objective of the primary SOC control unit is to offset $P_{B E S S}$ when it is deviated from 0 . Note that the primary SOC control unit must be able to measure $P_{B E S S}$ instantaneously to react instantly, which means that the communication mechanism should not be used to avoid communication time delay. To this end, the primary SOC control unit should be installed physically in the proximity of the BESS thereby the primary SOC control unit can measure $P_{B E S S}$ directly. Hence, DG3 in Fig. 1 is determined as the primary SOC control unit since it is installed at the same bus where the BESS is installed.

\section{Secondary SOC Control}

The control scheme of the secondary SOC control DG unit is shown in Fig. 4 where $S O C_{d}$ is the SOC level with communication time delay, $S O C_{n o m}$ is the nominal value of the SOC, $K_{P}$ and $K_{I}$ are the proportional and integral gains, respectively, $f$ is the system frequency, and $m_{P S}$ is the droop coefficient.

The main objective of the secondary control unit is to restore the SOC at the certain value, which is $S O C_{n o m}$ in this case. The current SOC level is informed to the secondary SOC control unit through communication infrastructure (MGCC) so the time delay is occurred. The error between $S O C_{n o m}$ and $S O C_{d}$ goes into the proportional-integral (PI) controller to maintain the SOC level. The frequency droop is also added to the controller to enhance reliability. If the communication system operates normally, the frequency droop control will not be implemented since the frequency would be maintained at the nominal value. On the other hand, if the communication system fails, the BESS will be operated as droop control mode thereby the secondary SOC control unit can share the active power based on frequency deviation. DG1 and DG2 are determined as the secondary SOC control units.

\section{SimULATION RESULTS}

The proposed control method is modeled and simulated with MATLAB/SimPowerSystems to prove its effectiveness. It is compared with the conventional frequency droop control.
Three cases are tested in this paper. The load change, the

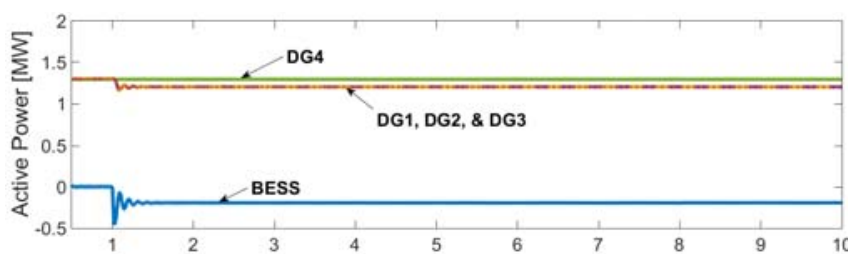

(a)

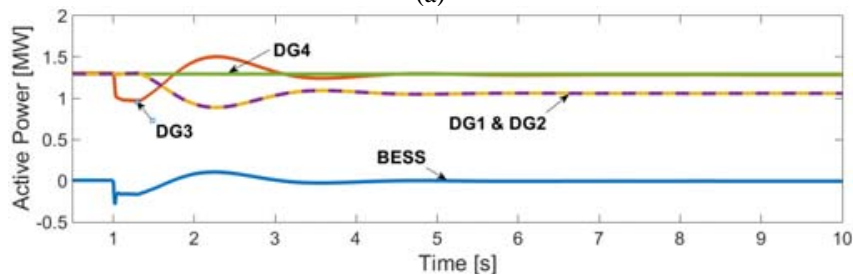

(b)

Figure 5. Active power for Case I. (a) Conventional method. (b) Proposed method.
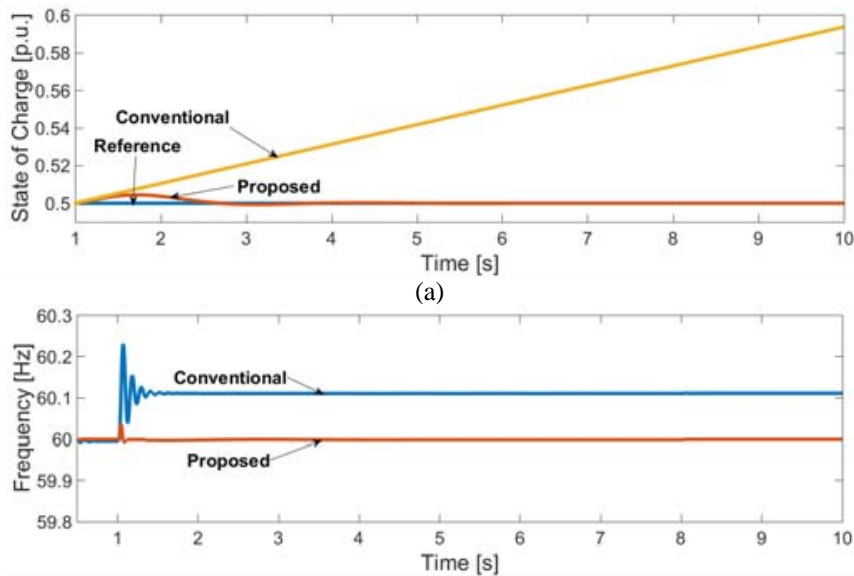

(b)

Figure 6. Comparison between conventional and proposed methods for Case I. (a) SOC. (b) Frequency.

intermittent output power change of uncontrollable DG unit, and the communication system failure during active power sharing are tested in Case I, II, and III, respectively. Typically, communication time delay can be in the order of 100-300 ms [17]. In this paper, we consider $300 \mathrm{~ms}$ of communication delay to account for the worst time delay. Since the total simulation time is about $10 \mathrm{~s}$, the capacity of the BESS is scaled down to $1 / 400$ of its original value (2 $\mathrm{MWh}$ ) to see change of the SOC clearly.

\section{A. Load Change-Case I}

In Case I, a sudden load change of $0.5 \mathrm{MW}$ is occurred at $1 \mathrm{~s}$ of the simulation time. Figs. 5 (a) and (b) present the active powers of the BESS and the DG units for the conventional droop method and the proposed method, respectively. In the conventional method, DG1-3 share the load change instantaneously. In the proposed method, the primary SOC control unit (DG3) shares the load change instantaneously and then $0.3 \mathrm{~s}$ (communication delay) after the load change, the secondary SOC control units (DG1 and 


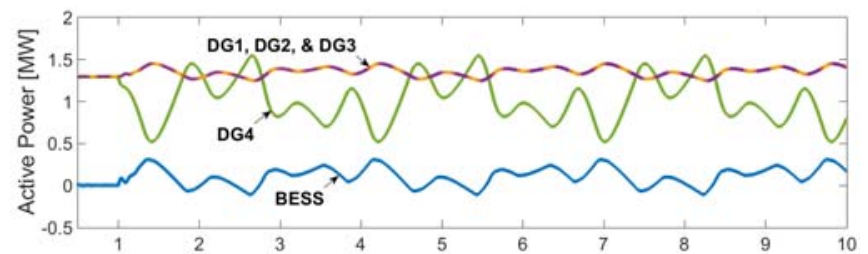

(a)

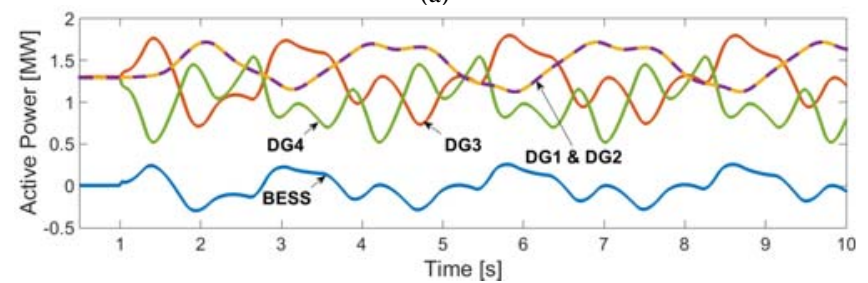

(b)

Figure 7. Active power for Case II. (a) Conventional method. (b) Proposed method.

DG2) share the load and restore the SOC to the certain value. Consequently, the output of the BESS is restored to 0. Figs. 6(a) and (b) presents the SOC and the frequency,

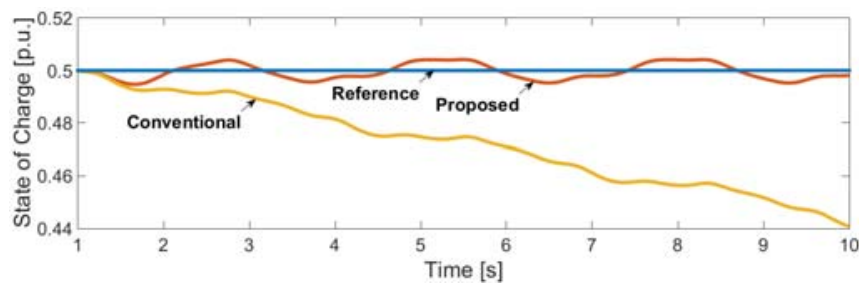

(a)

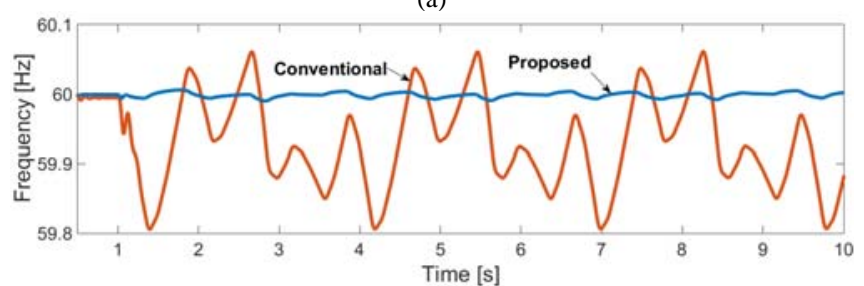

(b)

Figure 9. Comparison between conventional and proposed methods for Case II. (a) SOC. (b) Frequency.

respectively for both methods. As can be seen in Fig. 6(a), the SOC cannot be maintained at the desired value in the conventional droop method whereas it can be well maintained at the desired value (here, 0.5 p.u.) in the proposed method. Moreover, the frequency deviation is inevitable in the conventional method whereas the frequency is barely deviated from its nominal value while adopting the proposed method. In conclusion, the proposed method can prevent frequency deviation and maintain the SOC at the desired level after load change. Simultaneously, the active power sharing can also be implemented.

\section{B. Output Power Change-Case II}

Case II accounts for the case where the output of uncontrollable DG unit (DG4) fluctuates. The fluctuation starts at $1 \mathrm{~s}$. Figs. 7(a) and (b) shows active powers of the conventional method and the proposed method, respectively. As can be seen from Fig. 8(a), the SOC can be maintained at

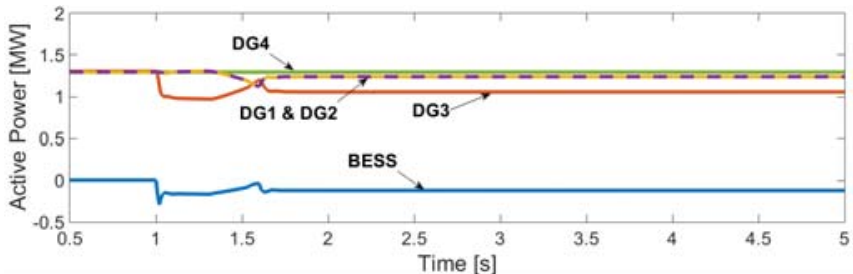

(a)

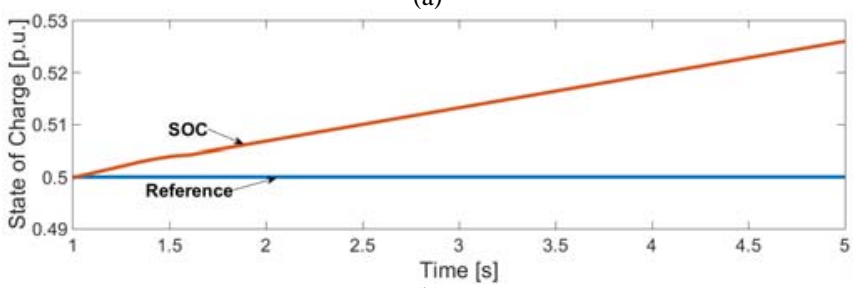

(b)

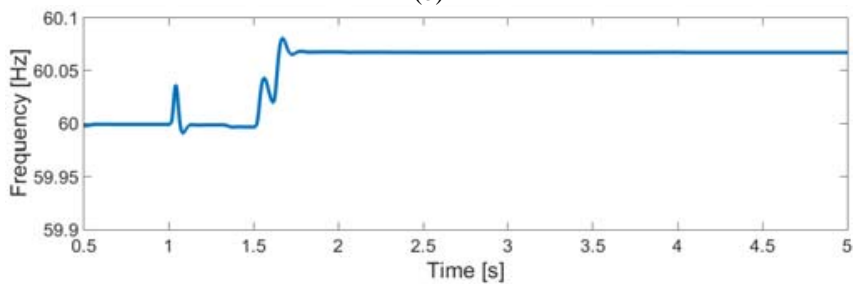

(c)

Figure 8. Active power, SOC, and frequency for Case III. (a) Active power (b) SOC. (c) Frequency.

the desired level in the proposed method whereas it cannot be in the conventional method. Moreover, the frequency also can be maintained at the nominal value in the proposed method as shown in Fig. 8(b) while it cannot be in the conventional method. Consequently, the proposed method performs as purposed for both situations of load change and output fluctuation in the perspective of maintaining the SOC and frequency.

\section{Communication System Failure - Case III}

The centralized control system has a disadvantage of reliability degradation due to the communication system failure. Hence, we proposed to add the frequency droop at the controller to enhance reliability. Case III accounts for the communication system failure. The situation is as same as Case I except that the communication system fails at $1.6 \mathrm{~s}$ of the simulation. Fig. 9(a) presents the active powers of the BESS and DG units. After the load change event at $1.0 \mathrm{~s}$, the active power is shared according to the proposed method based on the SOC. As the communication system fails at 1.6 $\mathrm{s}$, the active power is shared as same as the conventional method. The SOC cannot be maintained at the desire level as can be seen in Fig. 9(b). Also, the frequency cannot be maintained at the nominal value as shown in Fig. 9(c). Though the SOC and frequency cannot be maintained, the proposed method enhances the system reliability during the communication system failure with a change of BESS control mode. Note that the other DG units do not need any control mode change. 


\section{CONCLUSION}

This paper presents a novel method of the active power sharing for an islanded microgrid. Commonly, most of the conventional methods have been used the frequency droop control. However, the system frequency is inevitably deviated from its nominal value if the load is changed. An islanded microgrid is vulnerable to the frequency deviation since it has low system inertia. Hence, the active power sharing method without the frequency deviation is necessary for an islanded microgrid. To this end, we employed the BESS to constantly form the grid frequency without any droop control. However, the BESS has a capacity limitation which makes the BESS unable to operate consistently. To overcome the capacity limitation problem, other controllable DG units are adjusted to maintain the SOC at the certain level and to share the active power based on the SOC deviation. These DG units can be categorized as primary and secondary SOC control unit. The primary SOC control unit reacts instantly as the active power of the BESS deviates from 0 . The secondary SOC control unit receives the current SOC data from MGCC. Though sending SOC data has communication delay, the SOC can be well maintained at the desired level with the coordination of the primary SOC control unit. The communication system failure is also considered to enhance the system reliability. If the communication system fails, with the change of the BESS control mode, the system can be activated as same as the conventional droop-based microgrid. The effectiveness of the proposed method was proved by the simulation tested and modeled with MATLAB/SimPowerSystems.

\section{ACKNOWLEDGMENT}

This work was supported by the Global Excellent Technology Innovation (20132010101890) of the Korea Institute of Energy Technology Evaluation and Planning (KETEP), granted financial resource from the Ministry of Trade, Industry \& Energy, Republic of Korea.

\section{REFERENCES}

[1] L.-Y. Chen, Y.-T. Yin, T.-Y. Ho, and Y.-Z. Chen, "Sensitized solar cells via nanomaterials: A recent development in quantum dots-based solar cells,” IEEE Nanotechnol. Mag., vol. 8, pp. 16-21, Jun. 2014.

[2] N. Hatziargyriou, H. Asano, R. Iravani, and C. Marnay, "Microgrids,” IEEE Power Energy. Mag., vol. 5, pp. 78-94, Jul./Aug. 2007.
[3] B. Lasseter, "Microgrids [distributed power generation]," in Proc. IEEE Power Engineer. Soc. Winter Meet., Jan. 2001, vol. 1, pp. 146149.

[4] R. Lasseter, "Microgrids," in Proc. IEEE Power Engineer. Soc. Winter Meet., Jan. 2002, vol. 1, pp. 305-308.

[5] D. E. Olivares et al., "Trends in Microgrid Control," IEEE Trans. Smart Grid, vol. 5, pp. 1905-1919, Jul. 2014.

[6] F. Gao and M. R. Iravani, “A control strategy for a distributed generation unit in grid-connected and autonomous modes of operation,” IEEE Trans. Power Del., vol. 23, pp. 850-859, Apr. 2008.

[7] K. D. Brabandere, B. Bolsens, J. V. den Keybus, A. Woyte, J. Driesen, and R. Belmans, "A voltage and frequency droop control method for parallel inverters,” IEEE Trans. Power Electron., vol. 22, pp. 11071115, Jul. 2007.

[8] Y. Li, D. M. Vilathgamuwa, and P. C. Loh, "Design, analysis, and real-time testing of a controller for multibus microgrid system,” IEEE Trans. Power Electron., vol. 19, pp. 1195-1204, Sep. 2004.

[9] C. K. Sao and P. W. Lehn, "Autonomous load sharing of voltage source converters,” IEEE Trans. Power Del., vol. 20, pp. 1009-1016, Apr. 2005.

[10] T. L. Vandoorn, B. Meersman, L. Degroote, B. Renders, and L. Vandevelde, "A control strategy for islanded microgrids with DC-link voltage control,” IEEE Trans. Power Del., vol. 26, pp. 703-713, Apr. 2011.

[11] X. Yu, A. M. Khambadkone, H. Wang, and S. T. S. Terence, "Control of parallel-connected power converters for low-voltage microgridPart I: A hybrid control architecture,” IEEE Trans. Power Electron., vol. 25, pp. 2962-2970, Dec. 2010.

[12] A. Kahrobaeian and Y. A.-R. I. Mohamed, "Network-based hybrid distributed power sharing and control for islanded microgrid systems,” IEEE Trans. Power Electron., vol. 30, pp. 603-617, Feb. 2015.

[13] J. A. Peças Lopes, C. L. Moreira, and A. G. Madureira, "Defining control strategies for microgrids islanded operation," IEEE Trans. Power Syst., vol. 21, pp. 916-924, May 2006.

[14] I.-Y. Chung, W. Liu, D. A. Cartes, E. G. Collins, and S.-I. Moon, "Control methods of inverter-interfaced distributed generators in a microgrid system,” IEEE Trans. Ind. Appl., vol. 46, pp. 1078-1088, May/Jun. 2010.

[15] F. Katiraei, M. R. Iravani, and P. W. Lehn, "Micro-Grid autonomous operation during and subsequent to islanding process," IEEE Trans. Power Del., vol. 20, pp. 248-257, Jan. 2005.

[16] F. Katiraei and M. R. Iravani, "Power management strategies for a microgrid with multiple distributed generation units,” IEEE Trans. Power Systems, vol. 21, pp. 1821-1831, Nov. 2006.

[17] F. Milano and M. Anghel, "Impact of time delays on power system stability,” IEEE Trans. Circuits Syst. I, Fundam. Theory Appl., vol. 59, pp. 889-900. Apr. 2012. 